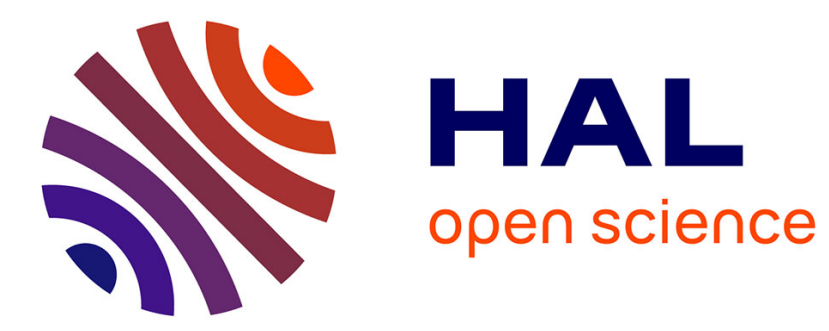

\title{
Être une fille, un gay, une lesbienne ou un.e trans au collège et au lycée
}

Johanna Dagorn, Arnaud Alessandrin

\section{To cite this version:}

Johanna Dagorn, Arnaud Alessandrin. Être une fille, un gay, une lesbienne ou un.e trans au collège et au lycée. Le sujet dans la Cité - Revue internationale de recherche biographique, 2015, Être à l'ecole aujourd'hui, 6, pp.140-149. hal-01255682

\section{HAL Id: hal-01255682 \\ https://hal.science/hal-01255682}

Submitted on 13 Jan 2016

HAL is a multi-disciplinary open access archive for the deposit and dissemination of scientific research documents, whether they are published or not. The documents may come from teaching and research institutions in France or abroad, or from public or private research centers.
L'archive ouverte pluridisciplinaire HAL, est destinée au dépôt et à la diffusion de documents scientifiques de niveau recherche, publiés ou non, émanant des établissements d'enseignement et de recherche français ou étrangers, des laboratoires publics ou privés. 


\section{Arnaud Alessandrin}

Sociologue, chercheur associé au Centre Emile Durkheim, Université de Bordeaux

\section{Johanna Dagorn}

Sociologue, chercheuse associée au LACES, Observatoire International de la violence à l'école, Université de Bordeaux

\section{"Etre une fille, un gay, une lesbienne ou un.etrans au collège et au lycée »}

«On doit aussi s'intéresser à ce qui ne se dit pas»(Les matins gris de la tolérance,Foucault,1977)

Résumé :Les travaux sociologiques autour de l'école menés depuis les années 70 démontrent que «l'indifférence aux différences » renforce les inégalités. Al'aune des approches de genre et de discrimination, cette « indifférenciation » contribue aussi à la discrimination des élèves non conformes aux normes de genre. A l'intersection d'une vision macrosociologique et micro, combinant des données statistiques et entretiens auprès des personnes discriminées, cet article propose une réflexion sur le sexisme, l'homophobie et la transphobie à l'école comme résultantes des normes sexuées, genrées, mais aussi sexuelles, et dévoile des résultats inédits d'une enquête de victimation.

\section{Introduction : de l'élève universel à l'individu genré.}

«Etre à l'école» a longtemps renvoyé à l'image d'un processus neutre et universelde construction des élèves, bien qu'excessivement local. Dans cette perspectives, dont on sait combien elle a pu invisibiliser la dimension de classes ou les tensions urbaines par exemple, les élèves n'ont longtemps pas eu de sexe, et par conséquent non plus de sexualité (Dagorn, 2014). Or, la citoyenneté contemporaine ne se satisfait plus uniquement des conceptions définitionnelles abstraites. Elle prend notamment corps autour de notions récentes comme la lutte contre les discriminations, à la jonction entre un processus de reconnaissance universel et particulier, celui-là même des minorités alors protégées. Pour le dire autrement, "être à l'école » devient une expérience qui se fractionne, non seulement en fonction des âges, mais également en fonction des caractéristiques singulières de l'élève, dont le genre et la sexualité 
font aujourd'hui pleinement partie (en termes de droit comme d'identité).A travers la construction du féminin et du masculin, les jeunes ne vivent donc pas la même expérience scolaire. Ainsi, les injonctions à la virilité impactent sur les libertés des filles et excluent, voire stigmatisent les garçons et filles en dehors de ce modèle (Joing-Maroye, Debarbieux, 2013).L'homophobie quant à elle s'inscrit durablement dans l'expérience scolaire des jeunes LGBT $^{1}$ (Chamberland, Richard, 2014). Toutefois, ce n'est que très récemment, en France tout $\mathrm{du}$ moins, que ces questions sont apparues sur les agendas politiques et dans les préoccupations de recherches. Aujourd'hui il nous est possible, àl'aide des dernières enquêtes concernant la perception du climat scolaire par les élèves dans l'institution ${ }^{2}$, de réfléchir sur la place des filles et des garçons à l'école, de la primaireau lycée, grâce aux dernières données concernant la perception du climat scolaire par les lycéen.ne.s. notamment. Dans ce contexte relevant du bien vivre ou du mieux vivre à l'école, la question du genre trouve toute sa place. Cette dernièrecomplexifie des débats concernant la domination masculine, en soulignant la manière dont certaines normes et polices de genrerestreignent la liberté et la participation scolaire des filles et garçons non conformes aux catégories de genre socialement construites.Pour restituer cela, tant sous une forme quantitative que qualitative,nous croiserons les enquêtes statistiques de victimation (Observatoire International de la violence à l'école, 2011, 2012 et enquête DMPV/OUIEP, 2014 ${ }^{3}$ avec les récits, observations et témoignages recueillis lors d'enquêtes sur l'expérience des discriminations ${ }^{4}$. Au total, nous esquisserons ici ce qui renvoie à l'expérience scolaire des jeunes LGBT et des filles, en prenant soin de souligner le continuum existant entre « sexisme», «homophobie » et «transphobie » (Alessandrin et Espineira, 2015).

\section{$I^{\circ} L^{\prime}$ expérience scolaire en proie au sexisme}

Très vite, l'enfant adopte des comportements conformes à son sexe d'appartenance et développe des représentations sur le masculin et le féminin (Dafflon-Novelle, 2010). Il identifie son appartenance sexuelle assez tôt, vers 1 an et demi (Blos, 1963), mais il ne s'identifie à un rôle social qu'au fur et à mesure de sa croissance et de sa socialisation. Dans ce contexte, chaque garçon, chaque fille, doit apporter les preuves qu'il, elle, est comme les autres garçons, les autres filles. Or, la construction de l'identité ne s'effectue pas dans les mêmes conditions en fonction des sexes. De nombreuses recherches ont par exemple montré que si les garçons sont poussés à explorer l'espace, à développer leur agressivité (dite positive) à travers les jeux de construction, de guerre notamment (Ruel, 2009). Les filles, quant à elles, sont davantage encouragées à investir les espaces de l'intime et du privé. Parallèlement, chaque garçon et chaque fille est contraint de construire son identité

\footnotetext{
${ }^{1}$ Lesbiennes, Gays, Bi, Trans.

${ }^{2}$ Observatoire International de la violence à l'école $(2011,2014)$, enquête SIVIS 2012 et DEP 2013.

3L'Observatoire Universitaire International Education et Prévention (OUIEP) de Paris 12 a pour objet l'étude de la prévention comme moyen de transformation des conditions d'éducation et comme outil de traitement d'un certain nombre de problèmes sociaux dans les champs de l'école.

${ }^{4}$ Arnaud Alessandrin, « Les bordelais.es face aux discriminations », Mairie de Bordeaux, 2015. Arnaud Alessandrin, Karine Espineira, Maud-Yeuse Thomas, «Tableau noir : les transidentités et l'école », Cahiers de la transidentité, 2014.
} 
personnelle en prenant position par rapport à des attentes sociales traditionnellement propres à son sexe. Parmi les stéréotypes associés aux garçons, on peut citer les suivants : affirmatif, agressif, ambitieux, aventureux, casse-cou, confiant ${ }^{5}$. "Au collège, les profs y disent que je m'affirme pas assez, que je devrais être moins réservé...Mais je suis bien comme ça, moi ! Je comprends pas! » Laurent, 13 ans, élève de 4eme.

Les enquêtes menées par l'Observatoire International de la violence à l'école primaire (E. Debarbieux, 2011) ${ }^{6}$ validentd'ailleurs la prévalence des agressions des garçons.A l'école primaire, comme au collège, comme à l'âgeadulte, le taux d'agressions à caractère sexuel et sexiste touche davantage les filles que les garçons. $8 \%$ des filles au collège déclarent avoir subi des violences à caractère sexuel à l'école (OIVS, 2012) et 7\% se déclarent victimes de voyeurisme, contre $5 \%$ pour les garçons. L'enquête menée auprès des lycéen-n-e-s ${ }^{7}$ avec les mêmes variables complétées par la fréquence des agressions et des items concernant l'homophobie vient confirmer l'approche genrée de ces agressions. En effet, certains garçons sont significativement plus victimes de violences à caractère sexuel répétées que les filles. Par exemple, $1,7 \%$ des garçons en ont été victimes plus de 5 fois dans l'annéede retraits forcés des vêtements contre $0,5 \%$ pour les filles (ci tableau ci-dessous).

\section{Depuis le début de l'année scolaire : a-t-on tenté de vous retirer un vêtement contre votre gré ? $^{8}$}

\begin{tabular}{|l|l|l|l|l|l|l|}
\hline $\begin{array}{c}\text { Retirer des vêtements } \\
\text { contre son gré } \\
\text { Genre de la victime }\end{array}$ & jamais & 1 fois & 2 fois & 3 fois & 4 fois & $\begin{array}{c}5 \text { fois } \\
\text { et plus }\end{array}$ \\
\hline Une fille & 95.7 & $1.2 \%$ & $0.3 \%$ & $0.2 \%$ & $0 \%$ & $0.5 \%$ \\
\hline Un garçon & 92.2 & $1.5 \%$ & $0.8 \%$ & $0.7 \%$ & $0.3 \%$ & $1.7 \%$ \\
\hline
\end{tabular}

Chiffres issues de l'enquêtenationale de climat et d'expérience scolaire auprès des lycéens,

Délégation ministérielle Prévention et lutte contre la violence à l'école, OUIEP, 2014

Aussi,2\% des garçons ont été victimes de voyeurisme au lycée au moins cinq fois au cours de l'année contre $1 \%$ pour les filles. Plus de $3 \%$ ont connu des épisodes répétés de cyber-violence à caractère sexuel dans l'année, contre $0,6 \%$ pour les filles.Ces éléments convoquent alors une autre dimension explicative, ne renvoyant pas uniquement au genre de l'individu mais plus probablement à sa sexualité, réelle, supposée, testée ou affirmée. L'analyse des discriminations de genre à l'école s'épaissit donc d'une nouvelle dimension.

\footnotetext{
${ }^{5}$ Qualificatifs stéréotypés tirés de Williams et Bennet (1975).

${ }^{6}$ Cette enquête montre que sur l'ensemble des agressions recensées à l'école, les garçons en sont victimes à plus de $60 \%$. Tout en étant les principaux auteurs puisqu'ils représentent plus de $80 \%$ des agresseurs identifiés. (Ces proportions sont identiques à celles de SIVIS, car même si le nombre d'incidents relevés dans ce type d'enquête est amplement sous-estimé, les proportions au sein de l'étude demeurent fiables, toutes choses égales par ailleurs).

${ }^{7} \mathrm{~N}=14916$, délégation ministérielle prévention des violences, OUIEP, 2014

${ }^{8}$ Le total en ligne ne fait pas $100 \%$ du fait des non réponses à cette question
} 


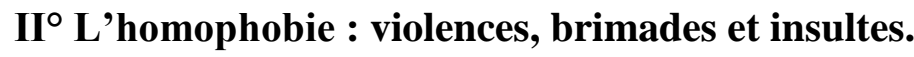

Ces violences sexuelles dont certains jeunes sont victimes instaurent souvent une position de domination genrée. Ainsi, le fait de « déshabiller de force », qui est un des items sexuels dont les garçons sont le plus victimes avec le voyeurisme, ${ }^{9}$ est un acte visant à dévaloriser les plus faibles et censé instaurer davantage de virilité au sein du groupe de pairs. Plus le harcèlement augmente, plus l'attaque contre la pudeur augmente (E. Debarbieux, 2011, p.28). Dans cette même étude, E. Debarbieux constate que plus de $70 \%$ des élèves victimes de harcèlement ont été victimes de voyeurisme. Il s'agit donc ici d'humilier sexuellement le plus faible, quel que soit son sexe, si on analyse ces violences de manière genrée. Ce type de violence est d'ailleurs majoritairement de type homophobe.Les chiffres de l'association «SOS Homophobie » sont sans appel : 59\% des agressions homophobes enregistrées en 2014 dans l'institution scolaire prennent la forme d'insultes, $21 \%$ d'agressions physiques et $12 \%$ de diffamations. C'est dire le poids et la dureté de l'homophobie pour les victimes. Laurent a 15 ans. Dans son lycée général "bien fréquenté » il entend souvent des insultes homophobes. Quelques fois, il a même assisté à des bousculades «contre des homos ». Egalement homosexuel il explique bien la peur d'en parler, de se montrer mais également de combattre 1'homophobie. "Les gens ils croient que c'est rien, que c'est pour rire, mais en fait quand tu vois ça t'as juste pas envie que ça t'arrive les insultes, les brimades. Donc en fait tu te caches un peu toi aussi. Et tu fais comme si t'avais rien vu ou rien entendu. »

C'est dans une répartition différenciée et hiérarchisée des rôles (Héritier, 2005) que va s'opérer une partie de l'homophobie sur la péjoration du féminin (ne pas être une fille, «une tapette ») et sur le fait d'être un « vrai gars » face au groupe des garçons. La socialisation des garçons dessine deux groupes bien distincts : ceux qui arrivent à montrer leur force, à être les plus forts, les plus virils ; et les autres qui risquent d'être déclassés dans la catégorie des soushommes, des «pédés ». Aux premiers «les médailles de virilité » (S. Ayral, 2011), aux autres, l'opprobre. Les démonstrations de force, les bagarres fréquentes, les insultes à caractère sexiste et homophobe constituent le quotidien de nombreux garçons au collège (V. Houadec, 2013). Et certains de ces jeux, tels le chat-bite ${ }^{10}$ montrent à quel point il est crucial de ne pas figurer dans la catégorie du féminin. Ici, sexisme et homophobie se conjuguent et dessinent une cartographie de la classe, du groupe de pairs, de la cours de récréation et des activités peri-scolaires, fortement marquée par l'hétérosexisme (Dayer et Alessandrin, 2015). Stéphanie a 13 ans. Dans son collège elle voit bien la manière dont la transgression des normes de genre a un coût. "Tu vois comment je m'habille? Et bien tu peux être certain qu'à la récré ou même après les cours, je traine pas avec les gens d'ici. J'parle pas de maquillage donc j'suis pas assez intéressante pour les filles de ma classe, et les mecs c'est des mecs quoi, ils restent en eux ».

L'homophobie reste toutefois une des "discriminations discriminées" (Teychenné, 2013). Pour preuve, parmi les incidents signalés par l'institution en $2012^{11}$, $11 \%$ sont clairement

\footnotetext{
${ }^{9}$ Relevé dans l'enquête d'E. Debarbieux, UNICEF, 2011.

${ }^{10} \mathrm{Il}$ s'agit de presser les parties génitales du garçon le moins rapide, voire le plus faible.

${ }^{11}$ Les chef-f-es d'établissement remontent au ministère les incidents relevés, qui sont communément nommés

"signalements".
} 
identifiés comme discriminants (ce chiffre ne varie pas depuis 5 ans). Parmi eux, $6 \%$ relèvent de sexisme, $4 \%$ de racisme et seulement $1 \%$ d'homophobie. C'est dire l'imbrication des discriminations, l'intersectionnalité qui les anime.

La lesbophobie ${ }^{12}$, la gayphobie, la biphobie en dépit des actions de prévention assurées par des associations, sont donc encore très prégnantes à l'école ${ }^{13}$. Le tableau ci-dessous met en évidence la relative banalité des propos homophobes en milieu scolaire.

Dans l'année scolaire, avez-vous été confronté à des insultes contre les gays et les lesbiennes $?^{14}$

\begin{tabular}{|l|l|l|l|l|l|l|}
\hline & Jamais & 1 fois & 2 fois & 3 fois & 4 fois & 5 fois et + \\
\hline Filles & $84 \%$ & $5.5 \%$ & $2.4 \%$ & $1.8 \%$ & $0.3 \%$ & $3.6 \%$ \\
\hline Garçons & $76.6 \%$ & $4.9 \%$ & $2.8 \%$ & $2.0 \%$ & $0.6 \%$ & $10.6 \%$ \\
\hline
\end{tabular}

Chiffres issues de l'enquêtenationale de climat et d'expérience scolaire auprès des lycéens, Délégation ministérielle Prévention et lutte contre la violence à l'école, OUIEP, 2014

Ces chiffres mettent bien en évidence la banalité des propos homophobes. Ainsi un garçon sur quatre a déjà entendu des propos homophobes durant l'année scolaire écoulée. Sans prendre en compte les angles morts de telles déclarations, on ne peut que souligner la permanence du vocabulaire homophobe à l'école.

\section{III $^{\circ}$ La transphobie : grande oubliée des discriminations en milieu scolaire.}

Si les transgressions de genre sont sanctionnées, la notion de transphobie devrait également faire son entrée dans les préoccupations scolaires ${ }^{15}$.En France tout du moins, les recherches sur cette question sont presque inexistantes. La première question que l'on entend lorsqu'on travaille sur ce sujet est « y a-t-il vraiment des trans à l'école ? ». Parler des élèves trans ce n'est pas seulement parler des transitions (même si cela existe), c'est également désencastrer ces dernières des parcours médicaux pour les faire entrer dans la complexité des parcours de vie.

Pourtant, outre-Atlantique singulièrement, la question n'a pas tardé à être posée. En 1996, Mildred Brown et ChloeRounsley publient le livre «le vrai soi » («True Selves: UnderstandingTranssexualism - For Families, Friends, Coworkers, and HelpingProfessionals ${ }^{16}$ ) dans lequel on retrouve de nombreux points relatifs à l'école (les chapitres 2 et 3 portant

\footnotetext{
${ }^{12} 39 \%$ des victimes déclarées dans l'enquête 2015 de SOS Homophobie sont des lesbiennes. Ce chiffre met l'accent sur une réalité peu évoquée, lorsqu'on appréhende le plus souvent l'homophobie comme synonyme de gayphobie.

${ }_{13}$ Ces associations alertent régulièrement sur l'isolement et la détresse de certains élèves victimes d'homophobie.

${ }^{14}$ Le total ne fait pas $100 \%$ car il existe pour cette question un certain nombre de « non réponses ».

${ }^{15} 21 \%$ des transsexuels ont arrêté leurs études à cause de la transphobie (étude du crips, 2007, citée par D. Latour, 2011)

16 Traduisez : «Etre soi :comprendre le transsexualisme pour les familles, les amis, les collègues, et les professionnels de l'aide».
} 
plus précisément sur l'enfance et l'adolescence) : «L'environnement scolaire impose souvent de redoutables épreuves aux enfants trans. Non seulement ils se sentent souvent rejetés dans la classe de cours, mais ils craignent aussi les périodes de vacances et pauses déjeuner » (p.43). C'est par exemple le cas de Bert qui témoigne la page suivante : «En sortant de l'école, je courrais jusqu'à la maison car j'avais peur de me faire attraper par mes camarades de classe » (p.44). En 2008, sort également le livre «The Transgender Child: A Handbook for Families and Professionals » de Stéphanie Brill et RachelePeper. Là encore, l'espace scolaire revient fréquemment. Dès les premières pages, le livre met l'accent sur les expériences violentes mais silencieuses vécus au sein ou aux alentours des établissements : "Quand ils sont étiquetés comme "gender-variants " [...] beaucoup d'enfants subissent des insultes verbales et des attaques physiques » (p.9). Plus récemment, en 2011, c'est le livre «Helpingyourtrangenderteen » qui est sorti aux Etats Unis (Krieger, 2011). Nous constations alors qu'une littérature existe et qu'elle n'est pas parvenue jusqu'à nous ${ }^{17}$.

Les recherches d'Elisabeth Meyer (2014) autour de la notion de «gendercreativspectrum » et de «gendercreativ kids », soulignent par exemple que dans les cours élémentaires aux EtatsUnis, $8 \%$ des élèves ne répondent pas strictement aux traditions des rôles de genre. Il en découle un régime de sanction vis-à-vis des transgressions de genre qui englobe les situations de sexisme, mais également d'homophobie et de transphobie. L'enquête du GLSEN (Gay, Lesbian and Straight Education Network) de 2005, nous rapporte que l'expression de genre est la troisième cause de harcèlement à l'école après l'apparence physique (le poids, la mode), l'orientation sexuelle réelle ou supposée, et avant l'ethnicité ou l'appartenance religieuse. L'expérience scolaire est donc une épreuve pour de nombreuses personnes LGBT. Isolement, craintes dans l'accès aux espaces scolaires genrés (toilettes, vestiaires), absentéisme: les conséquences de la transphobie à l'école sont nombreuses (Alessandrin, 2014) et marquent profondément la vie des individus concernés (Dayer et Alessandrin, 2015). L'enquête menée par H\&S (Homosexualité et Socialisme) et Le MAG (Mouvement d'Affirmation Gay) en 2010 apporte quelques éclairages chiffrés sur la question. Selon les associations, $69 \%$ des jeunes trans (de 16 à 26 ans) ont déjà pensé au suicide. Et si la majorité des répondants reconnaissent avoir bénéficié d'une bonne acceptation de leurs identités de genre au sein de l'école, 9\% (le double pour les $\mathrm{Mt}^{18}$ ) avouent avoir vécu des situations de rejet. L'enquête souligne alors que les personnes trans doivent composer avec l'institution et le milieu scolaire pour ne pas trop être en proie à des situations de transphobie. Une minorité arrive à imposer leur identité choisie à l'institution (13\% de l'échantillon). Au total la moitié seulement ont fait un coming-out auprès de leurs camarades ou de l'administration et $18 \%$ des situations recouvrent des insultes. Pour 7\% d'entre elles il s'agit de harcèlement, $6 \%$ d'exclusion, $5 \%$ de menaces et autant d'agressions physiques ${ }^{19}$.

Alors qu'elle est la grande oubliée des rapports, colloques et enquêtes sur les discriminations en milieu scolaire, la transphobie, de par les témoignages et les chiffres mis à jour, mèrite à

\footnotetext{
17 On trouve cependant quelques références aux textes suscités dans le fascicule $\mathrm{n}^{\circ} 2$ de l'association lyonnais Chrysalide : «La transidentité et les proches » (2010).

${ }^{18} \mathrm{Mt}$ : personne assignée garçon à la naissance

$\mathrm{Ft}$ : personne assignée fille à la naissance

19 Disponible sur:

http://www.hes-france.org/IMG/pdf/Rapport_Prelim_Enquete JeunesTrans_Avril2009_Vfinal.pdf
} 
notre sens la plus grande attention. Aux côtés des cas d'homophobies et de sexisme, la question des «bonnes pratiques » est alors posée.

\section{Conclusion :}

De nombreuses enquêtes le montrent, par le jeu, les interactions d'apprentissage ou l'humour, s'ancrent ouvertement des comportements sexistesinduisant une sous-estimation de la victimation $^{20}$. Ces mécanismes génèrent des tensions, voire des violences sexistes souvent minorées par les protagonistes qui sont les adultes, non formés à ce type de violences ${ }^{21}$, les garçons et les filles qui assimilent nombre de ces violences à un jeu, et par les victimes ellesmêmes. Ces dernières ont un seuil de tolérance trop élevé en raison de la banalisation de ces insultes. La construction du masculin et du féminin entraîne des processus de hiérarchisation des différences (Liotard, 2012). Ce ne sont donc pas les différences entre les sexes qui sont ici posées ou remises en question, mais (comme pour toute discrimination) les hiérarchies implicites ou explicites qui sont légitimées par la société.

Cela amène une réflexion plus générale sur la vision des femmes et des hommes dans notre société. Du coté des garçons, individuellement, les assignations et attentes sociales liées aux sexes laissent de côté et/ou stigmatisent tous ceux qui ne correspondent pas à ces modèles ; il ne s'agit pas que des homosexuels, des trans, mais aussi des hommes sans attribut viril affiché...D'ailleurs, ce n'est pas l'orientation sexuelle à proprement parler qui est ici en jeu, mais l'identité de genre (Alessandrin et Bellebeau, 2014) non-conforme à la norme du groupe.

Les choses ne sont pas figées : la construction identitaire est progressive, continue tout au long de la vie, jamais acquise une fois pour toute. Les actions en faveur de l'égalité et de la lutte contre toutes les discriminations ne pourront être pérennes qu'avec la mobilisation de toutes et de tous ; quel que soit le sexe, l'origine ou le milieu social d'appartenance.

\section{Bibliographie}

ALESSANDRIN, A., ESPINEIRA K, (2015). Sociologie de la transphobie. Bordeaux : MSHA.

ALESSANDRIN, A., DAYER C, (2015). Entre survisibilité et invisibilité : polices et transgressions de genre dans le contexte scolaire. Oser l'autre : altérité et éducabilité dans la France contemporaine (DUGAS E., FERREOL G. dir). Bruxelles : EME ed, 83-101.

\footnotetext{
${ }^{20}$ On peut ici parler de violence symbolique dans la mesure où il y a incorporation de la violence par les victimes (P. Bourdieu, 1970).

${ }^{21}$ Bien que des études récentes tendent à montrer un meilleur équilibre et une prise en compte par les enseignants. Certaines recherches montrent que les garçons ne sont plus autant l'objet d'un traitement préférentiel de la part des enseignants, même si la parole continue à être plus facilement prise par ces derniers (C. de Boissieu, 2009, A. Jarlégan, 2011).
} 
ALESSANDRIN, A. (2014). Apprendre en cis-scolarité. Pour en finir avec la fabrique des garçons (AYRAL S., RAIBAUD Y. dir). Bordeaux : MSHA, 269-287.

ALESSANDRIN, A., E-BELLEBEAU B. (2014). Genre! L'essentiel pour comprendre.Paris : Des ailes sur un tracteur.

AYRAL S., (2011). La fabrique des garçons : sanctions et genre au collège, Presses Universitaires de France.

BLOS P., (1963), Les Adolescents. Essai de psychanalyse, Paris, Stock.

BOISSIEU de (2009), Sexes et genres à l'école maternelle. Un essai de modélisation du concept de «genre scolaire », Paris, Recherches et Educations.

BRILL, S., PEPPER, R. (2008). The transgenerchild. New-York :Cleisspress.

BROWN, M., ROUNSLEY, C. (2008). True Selves : Understanding transsexualism. JosseyBass ed.

DAGORN, J. (2014) Ecole du genre ou genre à l'école ?, Genre ! l'essentiel pour comprendre (ALESSANDRIN A, E-BELLEBEAU B. dir). Paris : des ailes sur un tracteur, 150-153.

DAFFLON-NOVELLE, A. (2010). Pourquoi les garçons n'aiment pas le rose ? Pourquoi les filles préfèrent Barbie à Batman ?Perception des codes sexués et construction de l'identité sexuée chez des enfants âgés de 3 à 7 ans, Genre et socialisation (ROUYER V. dir), Paris : Erès, 25-40.

DAYER, C. (2014). Sous les pavés le genre : hacker le sexisme. Genève : L'aube.

DEBARBIEUX, E. (2011).A l'école des enfants heureux...enfin presque. Paris : UNICEF.

DURU-BELLAT, M. (2002).Les inégalités scolaires. Paris : PUF.

FOUCAULT, M. (1977). Les matins gris de la tolérance, Dits et écrits, tome 2. Quarto Gallimard, 269-271.

HERITIER, F. (2005). Hommes, femmes : la construction de la différence. Paris : Le pommier. 
HOUADEC, V.(2013),Le genre et les modèles amoureux dans la littérature de jeunesse : éléments de compréhension de l'éducation sentimentale des jeunes en France. Thèse de doctorat. Université de Toulouse.

JARLEGAN, A., TAZOUTI, Y. (2011), Les pratiques parentales vues par les enfants. Précarités et éducation familiale (ZAOUCHE C. dir). Paris : Eres. 135-139.

JOING-MAROYE, I., DEBARBIEUX, E. (2013). Violences de genre et violences sexistes à l'école (dir), Recherches et éducations, nº 8 \& 9.

KRIEGER, I. (2011). Helping your transgender teen.New-Haven :Genderwise press.

LATOUR, D. (2011). Des élèves trans à l'école des garçons et des filles, Cahiers pédagogiques, 487, 19-20.

LIOTARD, P. (2012).L'éducation du corps à l'école. Mouvements, normes et pédagogies (1881-2011), Editions A.F.R.A.P.S..

MEYER, E. (2014), Supporting transgender and gender creativyounth. New-York : Peter Land publishing.

RICHARD, G., CHAMBERLAND, L. (2014) Violences homophobes, violences transphobes. Les cahiers de la transidentité (ALESSANDRIN A., ESPINEIRA K., THOMAS M-Y. dir), $3,77-95$.

RUEL, S. (2009). La construction culturelle des sexes du point de vue des enfants. L'enfant, sujet actif dans le processus de socialisation et de formation des identités de genre. Thèse, Caen, Université de Caen Basse-Normandie. 